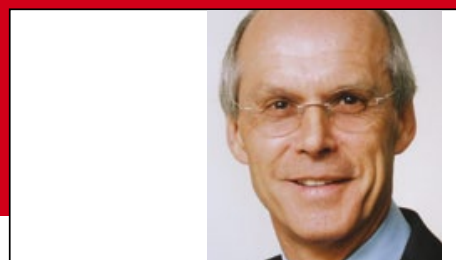

Prof. Dr. med. H. S. FüeßI

Isar-Amper-

Klinikum,

KI. München-Ost, Haar

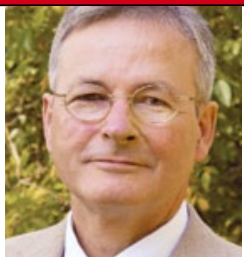

Prof. Dr. med. E. Fritschka

AHB-Klinik

für Nephrologie und

Diabetologie

Bad Brückenau

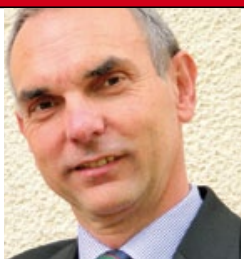

Prof. Dr. med. A. Gillissen

Klinik für Lungen und Bronchialmedizin, Kassel

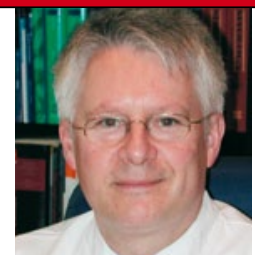

Prof. Dr. med. H. Reichmann

Klinikum Carl

Gustav Carus,

Dresden

\title{
Blinde Zeigefinger
}

\section{Die rektal-digitale Untersuchung scheint auf dem Rückzug zu sein: Medizinstudenten bekommen zu wenig Unterweisung, junge Ärzte haben bei der Untersuchung ein un- gutes Gefühl und Patienten lehnen sie nicht selten ab. Eine wertvolle manuelle diagnostische Methode droht verloren zu gehen.}

- 652 akademische Lehrer, Fach- und Assistenzärzte sowie Medizinstudenten im letzten Studienjahr von vier amerikanischen Medical Schools wurden mittels Fragebogen zu ihrer Praxis der rektaldigitalen Untersuchung (DRU) befragt.

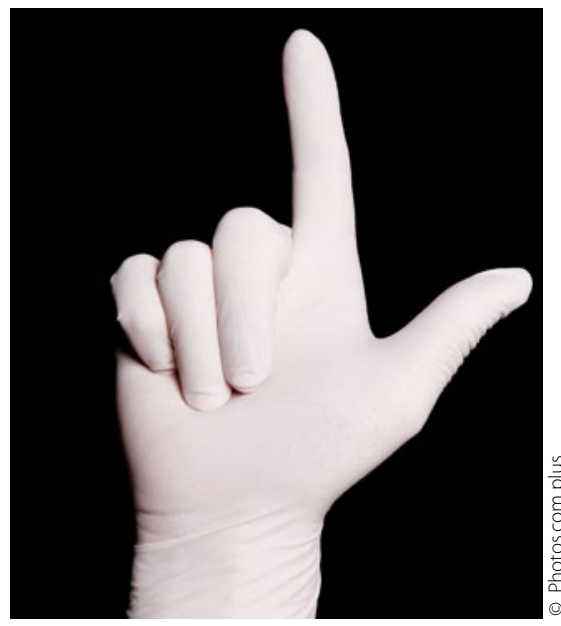

Kein Vertrauen mehr in den Zeigefinger.
Bei den Hochschullehrern und den Fachärzten handelte es sich um Allgemeinärzte, Allgemein-Internisten und Gastroenterologen.

Im Jahr vor der Befragung hatten die Teilnehmer $41 \pm 106$ DRU vorgenommen. Die Verteilung war erwartungsgemäß sehr ungleichmäßig. Die mediane Anzahl von Untersuchungen pro Jahr betrug nur 10, allerdings entfielen auf nur zehn Ärzte 500 und mehr Untersuchungen pro Jahr. 31\% der Befragten gaben an, sie würden sich mit der Untersuchung völlig sicher und wohl fühlen, $34 \%$ hatten einigermaßen Zutrauen zu ihren diesbezüglichen Fähigkeiten, 26\% gaben ein gewisses Unwohlsein an und $10 \%$ fühlten sich damit überhaupt nicht wohl.

Als wichtigste Gründe für die NichtDurchführung einer DRU wurden von $48 \%$ unspezifische Angaben gemacht, $44 \%$ scheuten die Untersuchung wegen einer möglichen Verletzung der Intimsphäre des Patienten, 40\% hielten die Untersuchung für zu invasiv und $36 \%$ gaben an, dass diese Untersuchung ohnehin vor der Koloskopie durchgeführt würde. Nicht selten hatten Ärzte erlebt, dass Patienten die Untersuchung ablehnten, wobei sich hier ein deutlicher Zusammenhang zwischen Fachgebiet bzw. Zutrauen und Erfahrung ergab. Gastroenterologen hatten das nur bei 4,3\% ihrer Patienten erlebt, Medizinstudenten bei $9,7 \%$ und Allgemeinärzte sogar bei $13,8 \%$. Für diese hohe Quote sind möglicherweise auch USA-spezifische Faktoren verantwortlich, wo man die Analregion noch stärker tabuisiert als in Europa.

\section{- R. K. Wong et al.}

The digital rectal examination: a multicenter survey of physicians' and students' perceptions and practice patterns. Amer. J. Gastroenterol. 2012; 107: 1157-1163.

\section{Kommentar}

Es wäre schade, wenn eine wichtige und in vielerlei Hinsicht sehr aussagekräftige Untersuchung nicht mehr gelehrt und kaum mehr angewandt würde und so in allgemeine Vergessenheit geriete. Für die Diagnose so verschiedener Erkrankungen und Funktionszustände wie Prostatahyperplasie und Prostatakarzinom, entzündliche Erkrankungen im Bereich des kleinen Beckens, Rektozelen, Raumforderungen im Rektum, und die Beurteilung einer fäkalen Impaktierung oder der Sphinkterfunktion in Ruhe und bei willkürlicher Kontraktion gibt es keine Untersuchung, die mit so geringem Aufwand so viele Aussagen machen kann. 